livraisons

d'Histoire

de l'Architecture

\section{Livraisons de l'histoire de l'architecture}

$31 \mid 2016$

La source photographique dans la pratique de l'historien de l'architecture

\title{
La photographie comme source pour une étude de l'architecture du Sacré-Cœur et de l'urbanisme de la butte Montmartre
}

Photos as historical sources for studying Sacré-Cour's architecture and butte of Montmartre's urbanism

Die Fotografie als Quelle einer architekturgeschichtlichen Studie von Sacré-

Cour und der Stadtplanung auf dem Montmartre-Hügel

\section{Jacques Benoist}

\section{(2) OpenEdition}

Journals

Édition électronique

URL : http://journals.openedition.org/lha/605

DOI : 10.4000//ha.605

ISSN : 1960-5994

\section{Éditeur}

Association Livraisons d'histoire de l'architecture - LHA

\section{Édition imprimée}

Date de publication : 14 juillet 2016

Pagination : 113-125

ISSN : 1627-4970

Référence électronique

Jacques Benoist, «La photographie comme source pour une étude de l'architecture du Sacré-Cœur et de l'urbanisme de la butte Montmartre », Livraisons de l'histoire de l'architecture [En ligne], 31 | 2016, mis en ligne le 14 juillet 2018, consulté le 19 avril 2019. URL : http://journals.openedition.org//ha/605 DOI : $10.4000 /$ /ha. 605

Ce document a été généré automatiquement le 19 avril 2019

Tous droits réservés à l'Association LHA 


\title{
La photographie comme source pour une étude de l'architecture du Sacré-Cœur et de l'urbanisme de la butte Montmartre
}

\author{
Photos as historical sources for studying Sacré-Cour's architecture and butte of \\ Montmartre's urbanism \\ Die Fotografie als Quelle einer architekturgeschichtlichen Studie von Sacré- \\ Cour und der Stadtplanung auf dem Montmartre-Hügel
}

Jacques Benoist

1 Deux mille photographies environ concernant le Sacré-Cœur et la butte Montmartre existent dans le fonds d'archives du diocèse de Paris et dans d'autres, conservés dans les institutions en rapport avec ces deux lieux historiques. En tout, on compte une vingtaine de collections. Je les ai répertoriées tout au long de mes travaux de recherches dont je rends compte en partie à travers cet article ${ }^{1}$. Je ne ferai que mentionner les archives photographiques sans chercher ni à les traiter ni à les énumérer: cela pourrait certainement faire l'objet d'une autre étude.

2 En particulier, j'ignore le reportage exécuté par Édouard Durandelle et Albert Chevojon, respectivement photographe et opérateur-tireur, durant une trentaine d'années sur le chantier de l'église dite du Vœu national au Sacré-Cœur de Jésus, un chantier qui, précisons-le, fut effectué à la demande d'un comité de construction présidé par les archevêques de Paris de 1872 à $1963^{2}$. Je l'ai déjà étudié dans mes précédentes publications. Claude Baillargeon l'a exploité et présenté dans un article paru en 1995 dans l'ouvrage collectif que j'ai dirigé dans le cadre de la Délégation à l'action artistique de la Ville de Paris, animée par Béatrice de Andia ${ }^{3}$.

3 Dans cette nouvelle étude, je présenterai quatre interactions entre photographies et textes qui ont toutes un rapport avec un bâtiment et/ou un site et leur histoire. La première présente Montmartre, haut lieu de naissance de la Commune de Paris, le 18 
février 1871, et le Sacré-Cœur, voulu le 2 décembre 1870 à Poitiers. La seconde interaction rend compte du monument funéraire Legentil, où reposent les cœurs des fondateurs du Sacré-Cœur. La troisième présente le cas du reportage photographique fait par la Conservation des objets d'art religieux et civils (COARC) de la Ville de Paris en 1985. Enfin, la quatrième présente la conception du "Vieux Montmartre ", selon Claude Charpentier, dans les années 1950 et 1960 : une conception dont l'aménagement de la Butte dépend encore aujourd'hui.

\section{«Photographies d'histoire »}

4 L'histoire du Sacré-Cœur comprend l'étude du choix de son lieu d'implantation, au sommet de la butte Montmartre. Sur le haut de cette colline se trouvaient des canons entreposés dans le champ des Polonais, au niveau du chevet de l'église Saint-Pierre, et que le gouvernement souhaitait soustraire aux Parisiens pour se prémunir contre d'éventuelles insurrections. La maison de la veuve d'Eugène Scribe (1791-1861), dramaturge français, se situait au même endroit, au 6, rue des Rosiers/Chevalier de La Barre, là où seraient morts successivement, en fin de journée, le 18 mars 1871, autrement dit le premier jour de la Commune, Claude-Martin Lecomte (1817-1871) puis Jacques Léon Clément-Thomas (1809-1871), tous deux généraux. Ce même lieu correspondrait aujourd'hui à l'emplacement du clocher du Sacré-Cœur et à l'entrée du carmel de Montmartre, au 34, rue du Chevalier de la Barre. Tous ces événements ont été doublés, en quelque sorte, par l'exécution d'Eugène Varlin (1839-1871) ${ }^{4}$, militant socialiste et membre de la Commune, le 28 mai 1871, dans un lieu de Montmartre qu'il faudrait encore déterminer.

Dans cet article, je ne me prononcerai pas sur les événements qui se sont produits au Château-Rouge et au 6, rue des Rosiers. Néanmoins, je remarque qu'une fois ces événements survenus, les histoires de la Butte, du Sacré-Cœur, de la Commune et de sa répression s'entrecroisent. Ils ont fait l'objet de nombreux commentaires. Ils ont été illustrés par autant d'images, devenues "mythiques». Mais à quel genre littéraire ces textes et ces visuels appartiennent-ils ? Et comment y démêler la fiction, le fictif et le faux

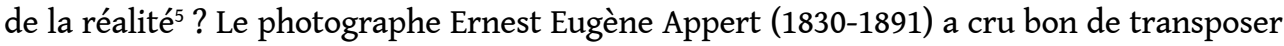
les événements marquants de la première journée de la Commune à la façon d'une peinture. Ce faisant, il a monté et composé une photographie, un photomontage des lieux, avec une mise en scène juxtaposant et formalisant les deux mises à mort ${ }^{6}$. N'est-il pas un « photographe d'histoire » comme il y a eu des peintres d'histoire?

Un éditeur dont les initiales sont G. C. A. a signé et diffusé ce photomontage pendant des décennies ${ }^{7}$. Il est devenu iconique de la Commune. Je dispose d'un de ces exemplaires (ill. 1), ainsi que d'une autre carte postale signée «C.P.» et présentée comme étant un « document historique » (ill. 2). L'histoire de ces deux éditeurs reste à écrire pour rendre compte de l'imaginaire dans l'histoire de Montmartre et du Sacré-Cœur 8 . 


\section{1 : l'exécution du 18 mars 1871}

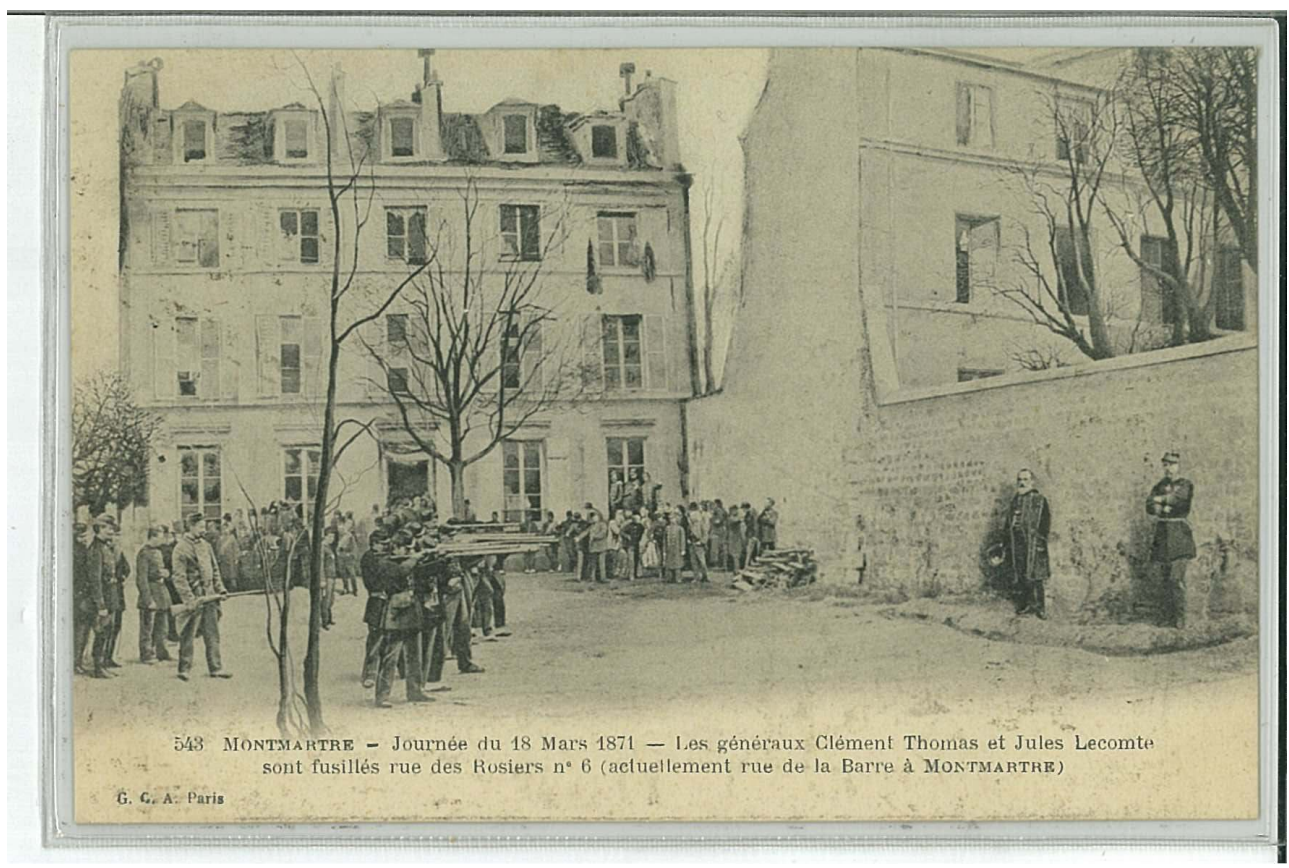

Photomontage. Carte postale, 105 × 155 mm, n 543, série « Montmartre », éditée à Paris par G. C. A (c) Coll. Jacques Benoist

\section{2 : l'exécution du 18 mars 1871}

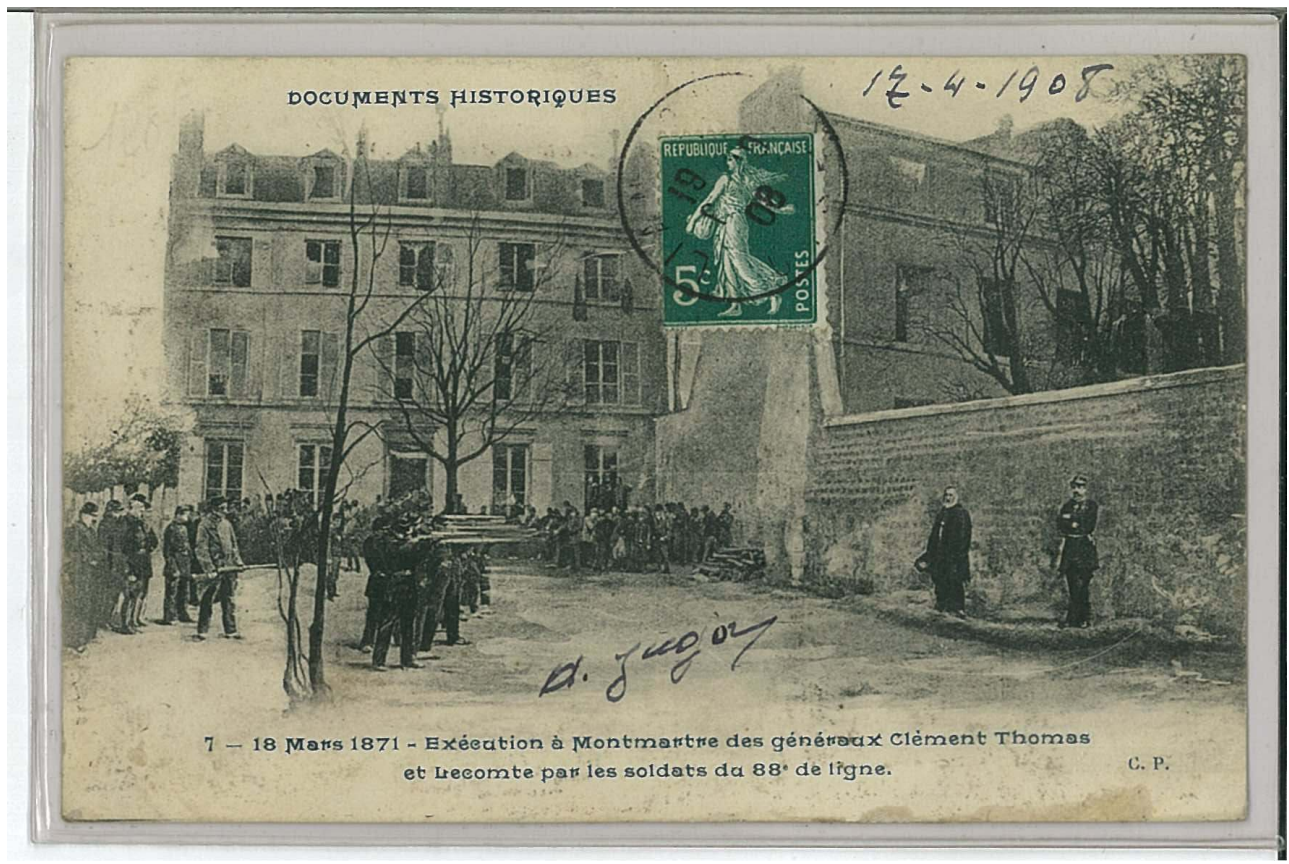

Photomontage. Carte postale, $105 \times 155 \mathrm{~mm}, \mathrm{n}^{\circ}$ 7, série " Documents historiques », éditée à Paris par C. P., 1871-1908.

(c) Coll. Jacques Benoist 


\section{Un cœur peut en cacher un autre !}

7 Un monument funéraire est dédié à Alexandre-Félix Legentil (1821-1889), initiateur du Voeu national à l'origine du Sacré-Coeur de Jésus en 1873. Il se situe dans la chapelle des morts de la crypte du Sacré-Cœur. Il se compose d'une colonne et d'une urne. Le tout est placé devant un texte de présentation, à destination des visiteurs. Legentil souhaitait que son cœur reposât dans l'église qu'il avait lui-même dédiée au cœur de Jésus à Montmartre : le Sacré-Coeur (ill. 3). Son épouse, Marie Legentil (1827-1920), née Marcotte, a veillé personnellement à la construction du monument funéraire de son époux. Cela se fit non sans mal, et avec du retard. Jean Hulot (1876-1959) en a été l'architecte. Le monument a été inauguré en $1921^{9}$. Depuis cette date, les visiteurs peuvent lire son explication sur une plaque déposée. Constatons que les photographies du monument, très répandues dans les guides touristiques, présentent toutes cette même plaque explicative, et lui confèrent leur autorité, si bien que personne ne l'a jamais remise en question jusqu'à aujourd'hui. Pourtant, cette plaque est inexacte. J'ai en effet découvert, dans une correspondance de Marie Legentil, qu'elle souhaitait qu'il en soit de même pour son propre cœur. J'ai alors réexaminé le monument funéraire, au-delà de l'inscription de la plaque et de sa sculpture: on pouvait ouvrir l'urne sans dommages et j'ai pu constater qu'elle est vide (ill. 4) (ill. 5). J’ai aussi remarqué la présence de deux croix gravées dans le sol, une à gauche et une à droite du pied de la colonne: les deux cœurs doivent certainement reposer là. Depuis cette découverte, l'inscription en question n'a pas été modifiée et les photographies des guides colportent toujours la même information erronée. Cet exemple nous enseigne quel type de regard les photographes portent sur leur modèle: ils s'intéressent avant tout à l'objet d'art sculpté, tangible et positif. L'historien quant à lui accorde de l'importance au négatif, à l'invisible - à ce qui est «hors-champ» - pour mieux établir la vérité historique - autant qu'on puisse la concevoir; pour lui, textes et images doivent être vérifiés ensemble. Du reste, nous pouvons nous demander combien de temps il faudra encore attendre avant de voir un second panneau rejoindre le premier pour expliquer l'invisible et ainsi rétablir la vérité ! 
III. 3 : vue du monument funéraire d'Alexandre-Félix Legentil



Sacré-Cœur, Montmartre.

(C) Cl. Benoît Le Bretton, 2015 
III. 4 : ouverture de l'urne non scellée du monument funéraire Legentil

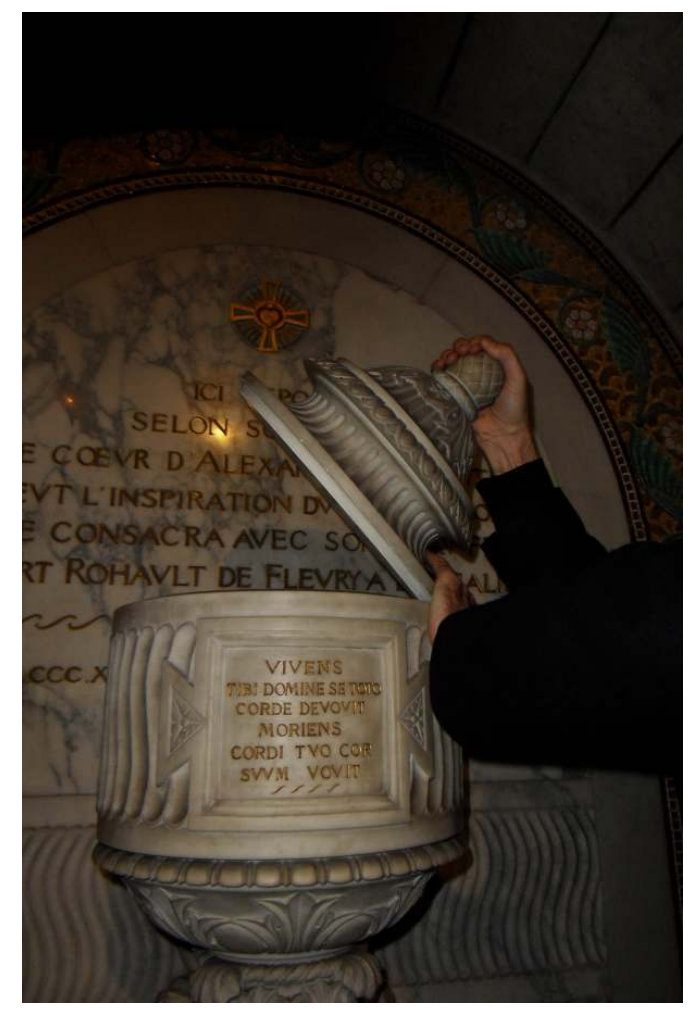

Sacré-Cœur, Montmartre.

(C) Cl. Benoît Le Bretton, 2015 
III. 5 : vue de l'intérieur de l'urne du monument funéraire Legentil

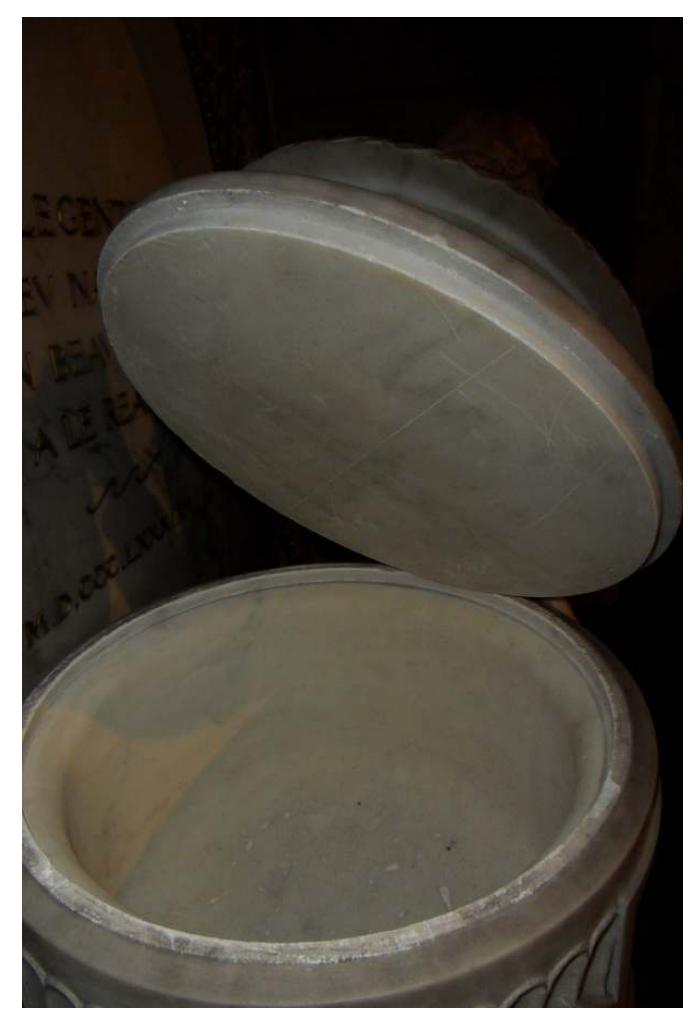

Sacré-Cœur, Montmartre.

(c) Cl. Benoît Le Bretton, 2015

\section{Les qualités et les limites d'un reportage photographique}

8 J'ai remarqué au moins deux absences dans le reportage d'un millier de photographies entrepris par Georges Brunel et Daniel Imbert en 1985 au titre de la Conservation des objets d'art religieux et civils de la Ville de Paris ${ }^{10}$ : d'une part, la statue Notre-Dame du Cap offerte vers 1960 par les Québécois pour décorer la chapelle souscrite par leurs pères (ill.6); et, d'autre part, le négatif encadré et éclairé de la photographie du tissu dit "suaire de Turin» (ill.7) ${ }^{11}$. Ces deux lacunes m'ont révélé quels étaient les préoccupations et les critères des deux conservateurs. Dès lors, j'ai appréhendé les reportages photographiques avec plus de précaution. Un tel travail, aussi exhaustif et légitime soit-il par le titre honorifique de son auteur, n'empêchera pas une personne avertie d'en constater les limites! Et, les historiens qui étudieront le devenir et l'état du Sacré-Cœur à la fin du $\mathrm{XX}^{\mathrm{e}}$ siècle à partir de ce reportage devront, je m'y engage, le compléter. Ils pourront faire mémoire de la chaire et du banc d'œuvre construits au début du XXe siècle par Lucien Magne, puis détruits au début des années 1960 par le recteur de l'époque, Maxime Charles. Ils pourront aussi parler des lampadaires et des lustres, qui ont connu le même sort. Ils devront certainement prendre des photographies de l'urne prétendument funéraire et des inscriptions du monument Legentil, mais avec circonspection - comme nous l'avons vu! S'ils veulent un état de la chasublerie du Sacrécœur, il leur faudra reconstituer la collection des éléments contemporains qui 
n'apparaissent pas dans le travail de Brunel et Imbert; un travail qui, par ailleurs, soulignons-le, est plein de qualités. Ils témoigneront d'une mise en scène du chœur sanctuaire qui a existé entre 1975 et 1995 environ et qui, depuis, a disparu. Cette enquête leur permettra de profiter de la redécouverte du cœur d'Anselme Batbie (1828-1887) ${ }^{12}$, qui avait été oublié dans un caveau depuis sa mort. Les historiens du futur prendront en compte, je l'espère, le chef d'œuvre de l'orfèvre parisien Joseph Chaumet (1852-1928), la Via Vitae (1894-1904, Paray-le-Monial, au musée du Hiéron), pour comprendre le SacréCœur dans le contexte historique de la Belle Époque. La sculpture a été réalisée pour le sanctuaire de Montmartre, mais n'y a jamais été exposée; elle a été déclarée trésor national en 2000.

\section{6 : statue de Notre-Dame du Cap (Québec)}

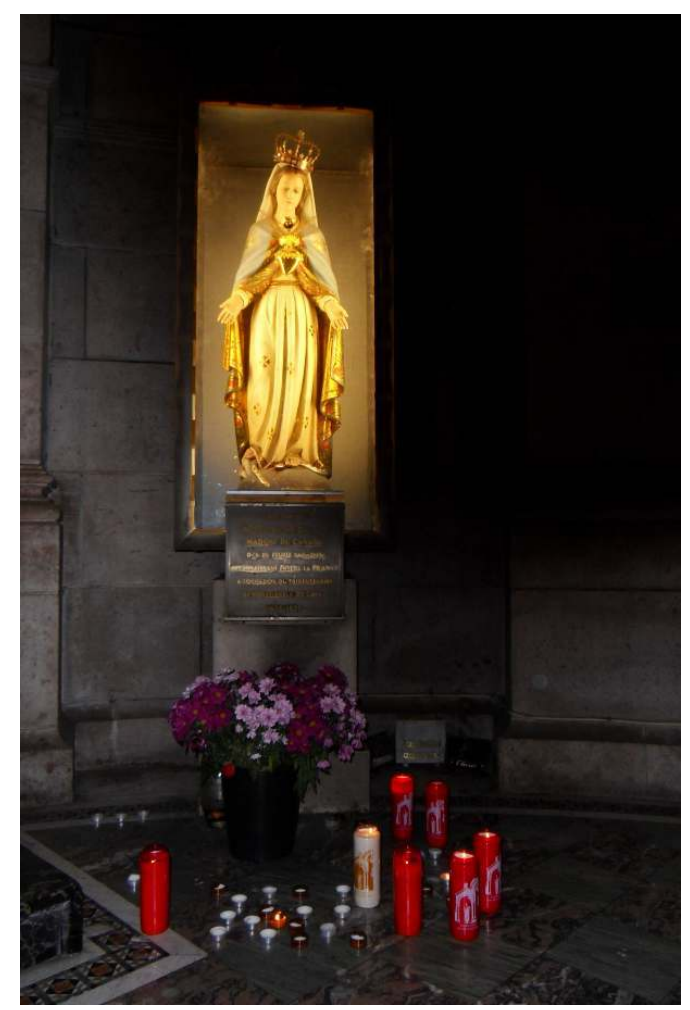

Chapelle Saint-Jean-Baptiste, Sacré-Cœur, Montmartre.

(c) Cl. Benoît Le Bretton, 2015 
III. 7 : monument de la face de Jésus selon le négatif de la photographie du tissu dit le « suaire de Turin»



Sacré-Cœur, Montmartre.

(c) Cl. Benoît Le Bretton, 2015

\section{Palissade ou grille?}

9 Aux côtés de la photographie d'histoire, de la photographie de guide touristique et de la photographie de reportage, la photographie d'urbanisme constitue aussi une source importante pour comprendre l'histoire de Montmartre. Qu'en est-il de l'urbanisme de la Butte? Ce quartier n'apparaît-il pas comme un quartier figé ? Aussi figé que les photographies de la Belle Époque - en particulier celles d'Eugène Atget -, figé comme les tableaux du peintre de l'École de Paris Maurice Utrillo (1883-1955), et, plus singulièrement, figé comme les souvenirs de l'architecte voyer de l'arrondissement des années 1950-1960 13 ? Cette fiction urbanistique est surtout l'œuvre de l'architecte urbaniste Claude Charpentier (1909-1995). En effet, l'action de ce dernier aurait été déterminante dans l'élaboration de la loi Malraux de 1962 relative à la protection des secteurs sauvegardés à Paris et dans la délimitation du périmètre du «Vieux Montmartre ", à l'image de celui du « Vieux Paris ».

10 Toutes ces informations sur l'urbanisme ont été regroupées par des historiens de la Ville de Paris puis reportées sur une «Pelle Starck ${ }^{14}$ ", actuellement placée au pied du château d'eau de la rue du Mont-Cenis, à côté de la place du Tertre. La mémoire, conservée dans la photographie, les peintures et les souvenirs, détermine tout un projet urbanistique.

11 La clôture du domaine du Sacré-Cœur constitue un autre bon exemple pour comprendre l'urbanisme de la butte Montmartre. Et, la photographie historique joue un rôle 
déterminant, là encore. Maxime Charles, recteur de 1959 à 1985, avait pour projet de mettre une grille le long de la rue Lamarck, entre la rue du Chevalier-de-La-Barre et la rue Muller/Maurice Utrillo, sur le côté Est du domaine (ill. 8). Elle devait remplacer une palissade qui datait de la fin du XIX ${ }^{\mathrm{e}}$ siècle. Elle aurait été dans la continuité de celle qui fermait alors les côtés Sud, Ouest et Nord. Pour Claude Charpentier, l'architecte urbaniste, il n'en était pas question, il le lui a interdit. Certes le terrain affecté au culte, au sens large, appartenait à la Ville, mais c'était à l'affectataire, en l'occurrence au recteur Maxime Charles, de le reconstruire et de le faire à l'identique! De la palissade restaurée dans les années 1960, on peut aujourd'hui encore admirer un vestige, au-dessus de l'édicule des toilettes, à l'angle Lamarck/Utrillo (ill. 9). Les proportions du bâtiment n'ont pas tellement changé depuis les années 1900, même si les visiteurs ont été multipliés par dix en un siècle. Je ne peux pas traiter l'histoire de la législation touchant la protection du patrimoine architectural, urbain et paysager de la Butte, depuis la liste de 1862 qui prend en compte Saint-Pierre de Montmartre jusqu'à aujourd'hui et aux ZPPAUP, ni entrer dans le détail des actions de l'architecte urbaniste Claude Charpentier. Je me contenterai de remarquer que ce sont certainement des photographies, des cartes postales et des tableaux qui ont dû conforter et matérialiser ses souvenirs d'enfance, lorsque celui-ci a entrepris de figer son Vieux Montmartre. Il faudrait traiter des façades, des alignements, des hauteurs des immeubles, des réverbères, du revêtement des chaussées et des trottoirs, des enseignes, des espaces verts publics et privés, du maintien d'un coefficient d'occupation des sols peu élevé et des reconstructions à l'identique, et ceci, au bout du compte, au service de la naissance et du développement d'un « parc à visiteurs » venus du monde entier. Quant à la palissade des années 1900, reconduite à l'identique dans les années 1960, elle a finalement été remplacée par une grille dans les années 2010 (ill. 10). La Ville a adapté les règlements voulus par Claude Charpentier ${ }^{15}$.

\section{8 : l'angle sud-est du domaine du Sacré-Cœur, angle Lamarck Muller-Maurice Utrillo vers 1900}

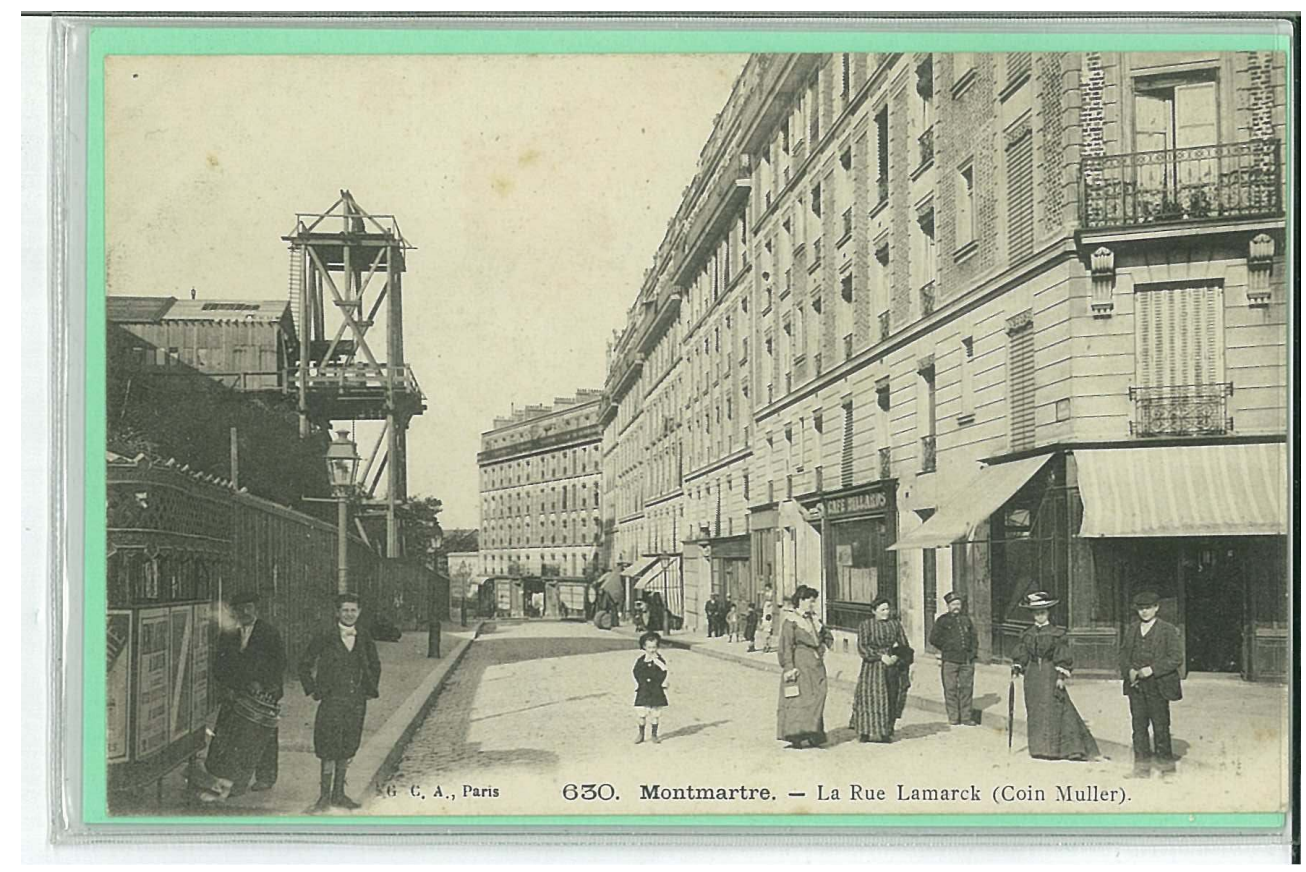

Carte postale, n 630, série « Montmartre », éditée à Paris par G. C. A.

(c) Coll. Jacques Benoist 
III. 9 : vue de l'angle sud-est du domaine du Sacré-Cœur, angle rue Lamarck, rue Muller/Maurice Utrillo

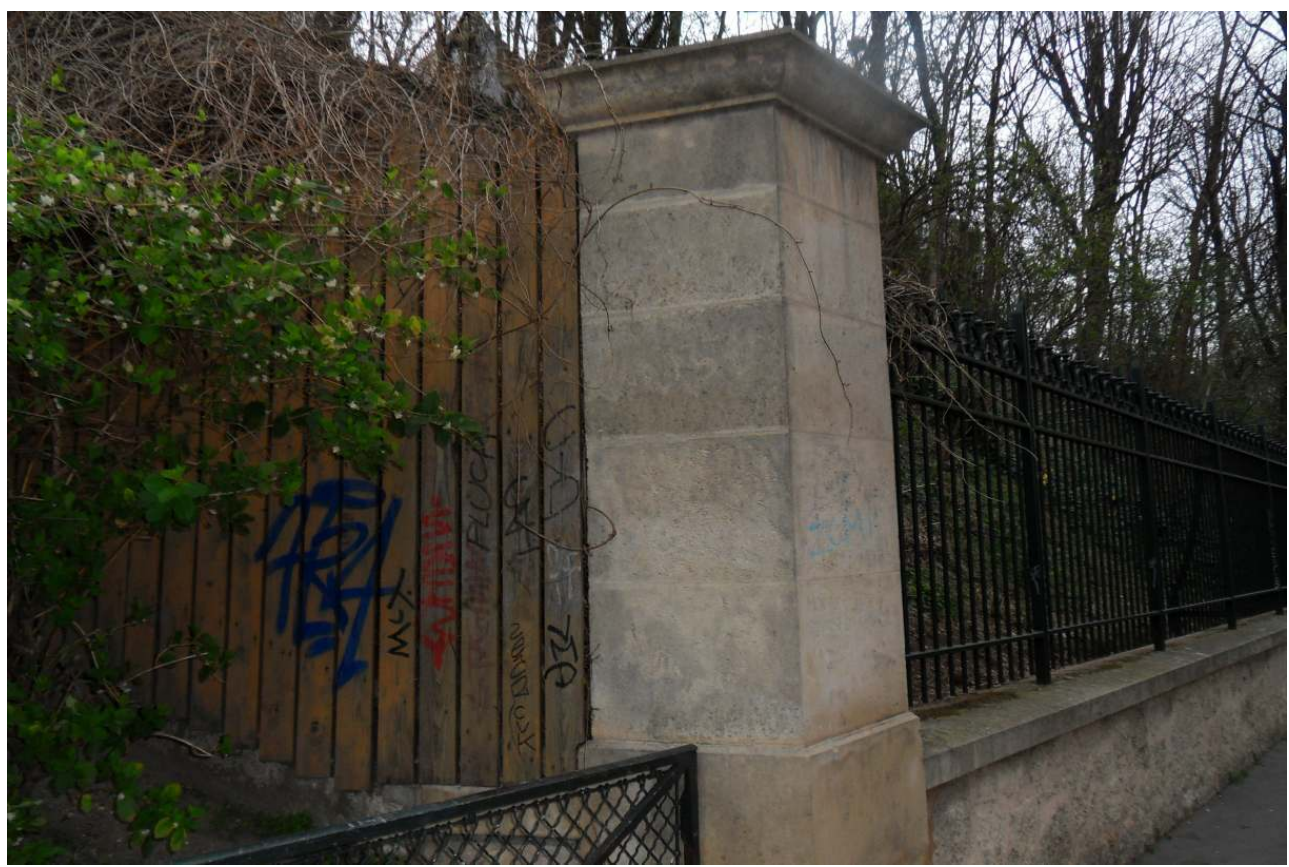

Prise en 2015, on y voit un reste de palissade et une grille.

(c) Cl. Benoit Le Bretton, 2015

III. 10 : angle du domaine du Sacré-Cœur, Lamarck/Muller-Maurice Utrillo

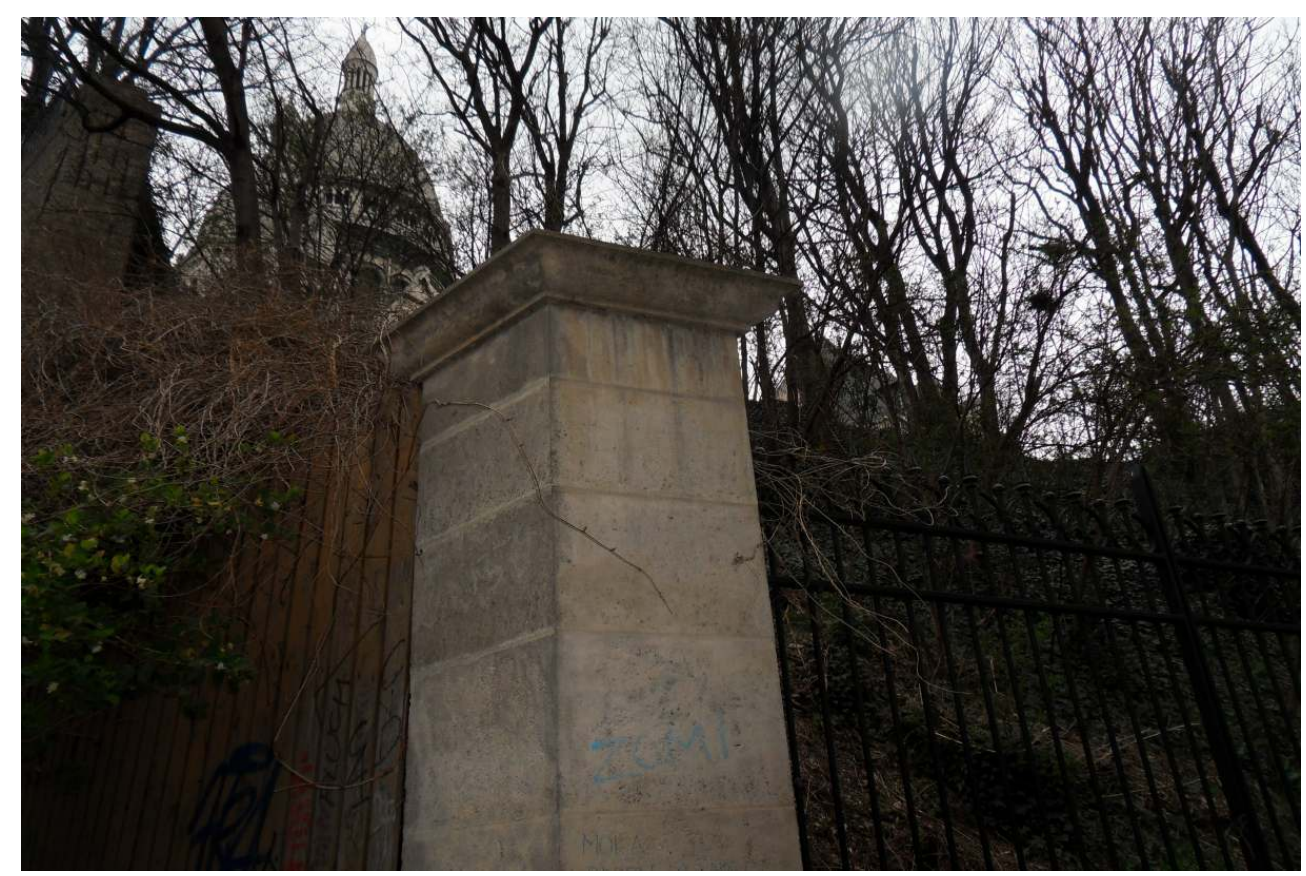

On y voit la palissade des années 1960 et la grille des années 2000

(c) Cl. Benoît Le Bretton, 2015

12 En conclusion de ces quatre réflexions sur l'interaction entre le terrain, les textes et les photographies, je présenterai un cas supplémentaire qui forme, selon moi, un appel à 
mener des recherches. Huit bas-reliefs sont actuellement déposés dans le comble du grand dôme du Sacré-Cœur, lieu probablement inaccessible au public depuis les années 1950. Je n'ai pas réussi à les documenter. Les photographies, seules, les font connaitre au public pour le moment. Quelques privilégiés, compte tenu du plan Vigipirate, peuvent les admirer lors de visites guidées. Un travail de documentation de la butte Montmartre et du Sacré-Cœur reste donc à faire ; et j'invite tout chercheur désireux de se lancer dans une telle entreprise à prendre en compte les textes historiques et les images photographiques, ensemble, car elles se complètent pour rendre compte d'un monument et d'un site, avec les qualités et les limites des unes et des autres. En d'autres termes, l'écriture-dessin (le texte), avec l'encre, et le dessin-écriture (la photographie), né de la lumière, se complètent et se compléteront au service de la connaissance par nos idées du réel, inépuisable et surprenant, sur la butte Montmartre et ailleurs.

\section{NOTES}

1. Je remercie de leur aide, M. Jean-Michel Leniaud, Mmes Charlotte Leblanc et Odile Boubakeur, MM. Jean-Charles Forgeret, Jean-Paul Bardet, Benoit Le Bretton et Jean-Pierre Gobit, et, dernièrement, Mme Anne-Cécile Schreiner.

2. Dates respectives de fondation du comité par Hippolyte Guibert, archevêque de Paris, et de sa dissolution par Maurice Feltin, archevêque de Paris, et Maxime Charles, recteur du Sacré-Cœur. Armand Floret (1917-1964) a été le dernier secrétaire général de 1961 à 1963. Voir la correspondance de Mme Isabelle Floret, sa petite-fille, avec l'auteur, en date du 28 novembre 2015.

3. Voir Elvire Perego, «Delmaet \& Durandelle ou la rectitude des lignes », Photographies, n 5, s. 1., printemps 1984, p. 54-75 ; et Jean Audouze, Gilles Portebos, Alain Hellou, Jean-Michel Gourden, Françoise Denoyelle, Le Studio Chevojon: une dynastie de photographes parisiens, Paris, Créaphis, 1994, 96 p.

4. Maurice Foulon, Eugène Varlin : relieur et membre de la Commune, Clermont-Ferrand, Éd. MontLouis, 1984, 244 p., p. 238-244 ; Maurice Dommanget, Eugène Varlin (1839-1871), « Les belles figures du prolétariat », Paris, La Ruche ouvrière, s. d., 48 p., p. 41-43.

5. Voir les travaux de l'Institut national pour la photographie contemporaine et ceux du Groupe d'étude et de recherche des médias spontanés, en collaboration avec l'université Paris VIII. Nouvelle biennale de mars 2000 à mars 2001. J'ai consulté la présentation de ces publications sous l'égide d'Alain Badiou.

6. Voir la bibliographie de l'article sur cette personnalité dans Wikipédia. Également l'article de Stéphanie Sotteau-Soualle, «Ernest Appert (1831-1890), un précurseur d'Alphonse Bertillon?», Criminocorpus, revue d'histoire de la justice, des crimes et des peines, 14 p. En ligne. Voir aussi les publications de Jacques Rougerie sur ce sujet particulier « Notes pour servir à l'histoire du 18 mars 1871 », qu'on trouve sur son site. Il n'est qu'une mention en trois mots de « l'assassinat des généraux » (dans le § 28 environ)! Qu'en penser?

7. Je ne sais pas quel est le lien entre Eugène Ernest Appert et «G. C. A., Paris ». A pour Appert ?

8. L'éditeur «G.C.A. » porte le numéro 26 dans la liste des éditeurs français de cartes postales, consultée sur Wikipédia. L'éditeur « C. P. » n’est pas mentionné. 
9. Voir le Bulletin du Vœu national, 1920, p. 24 et 1921, p. 84. Voir J. Benoist, Le Sacré-Cour, 1992, p. 43-44.

10. Ce projet s'est monté suite à ma suggestion, alors que j'étais responsable du nettoyage et de l'entretien du Sacré-Coeur, de 1981 à 1985.

11. Sur ce sujet, voir Luce Lebart, "L'homme du suaire. La première photographie de crime ", Images à charge. La construction de la preuve par l'image, Diane Dufour (dir.), Le Bal, Éd. Xavier Barral, mai 2015, 239 p., p. 61-79. Et l'article d'Odile Celier, «Suaire », Catholicisme, 1996, t. XIV, col.521-523, qui fait le point sur les auteurs favorables à l'hypothèse de l'authenticité et les auteurs critiques.

12. Anselme Batbie fut ministre des Cultes en 1873 , sous le premier gouvernement Albert de Broglie.

13. Voir le chapitre Montmartre pour cette notion de " quartier figé », Jacques Lucan (dir.), Paris des faubourgs, Paris, éd. du Pavillon de l'Arsenal, 1996, 224 p., 200 ill. noir et blanc et couleur.

14. Il s'agit de panneaux explicatifs en métal dessinés par le fameux designer Philippe Starck et édités par l'entreprise spécialisée en mobilier urbain JDecaux. Leur forme rappelle celle d'une pelle.

15. Voir Montmartre. Protéger et mettre en valeur, Mairie de Paris, 1996, A4 paysage, 40 p. Direction de l'aménagement urbain et de la construction. Sous-direction de l'environnement et de la réglementation. Service de l'espace public. 17, boulevard Morland, Paris IVe arrondissement. Conception et rédaction, agence Urbana, Anne Vourc'h.

\section{RÉSUMÉS}

Le rôle de la photographie comme source historique est souligné dans quatre exemples liés à l'histoire de la butte Montmartre et du Sacré-Cœur de Paris par le biais des travaux de l'auteur. Quelle est la place du photomontage signé «G. C. A. » présentant l'exécution des généraux le 18 mars 1871 ? Quelle est l'autorité des photographies qui reproduisent une image et une réalité non critiquées ? Comment voir les limites d'un reportage photographique qui paraît, de prime abord, être exhaustif? Enfin, qu'arrive-t-il quand ce n'est plus l'image qui est construite sur la réalité, mais la réalité qui est construite sur l'image?

In this article, photos as historical sources are highlighted with four examples which deal with the history of the mount Montmartre and the Sacré-Cœur in Paris and originate from the previous author's researches. What is the importance of the photomontage identified by initials "G.C.A. » representing the generals' execution March the $18^{\text {th }} 1871$ ? What kind of authority comes from photographs which reproduce both uncritical picture and reality? How can one see limits of a photo report, which seems to be at first sight exhaustive? What does happen when picture is no longer based on reality but, on the contrary, when reality is built from the picture?

Anhand von vier Beispielen auf dem Montmartre-Hügel soll in diesem Beitrag die Bedeutung der Fotografie als historische Quelle verdeutlicht werden. Ausgangspunkt ist die Frage nach der Rolle der mit den Initialen "G.C.A." unterzeichneten Fotomontage der Hinrichtung von Militärgenerälen während der Pariser Kommune am 18. März 1871. Welchen Wirkungsraum entfalten die kaum angezweifelten Bilder und welche Realität scheinen sie widerzuspiegeln? Wo liegen die Grenzen einer scheinbar umfassenden Fotoreportage? Und was passiert, wenn die 
Bezüge zwischen Realität und Abbildung zusehends verschwimmen und nicht mehr das Foto die Realität abbildet, sondern sie scheinbar erst erzeugt?

\section{AUTEUR}

\section{JACQUES BENOIST}

Jacques Benoist, né en 1946, est ecclésiastique, maître en théologie de l'Institut catholique de Paris, docteur en histoire des religions de l'université Paris IV-Sorbonne et élève diplômé de l'École pratique des hautes études, section des sciences historiques et philologiques. Il a publié Le Sacré-Cœur de Montmartre, t. I, La Spiritualité, l'art et la politique : 1870-1923 ; t. II, La Contestation: 1870-1990 ; t. III, Le Féminisme, l'urbanisme et le tourisme : 1870-1960, Paris, éd. de l'Atelier, 1992 et 2000, 1776 p. Il a également collaboré à la direction de l'ouvrage collectif, Un Vœu national au Sacré-Cœur de Jésus, Paris, Délégation à l'action artistique de la Ville de Paris, 1995, 262 p. Adresse électronique : jacques@benoist.net 